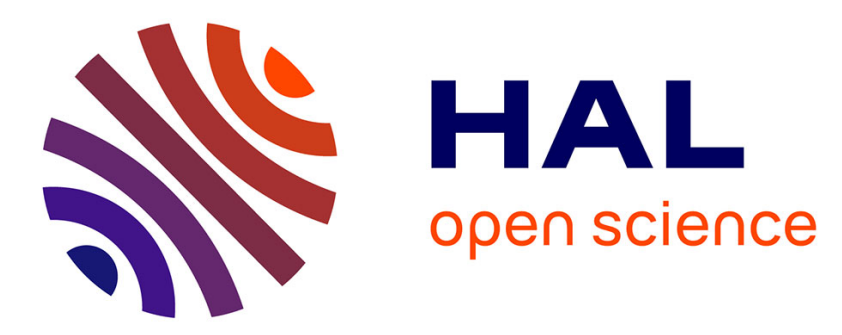

\title{
The $\gamma-\epsilon$ Martensitic Transformation: A Model for Stress Induced Variant and its Interaction with Grain Boundary
}

\author{
G. Guénin
}

\section{- To cite this version:}

G. Guénin. The $\gamma-\epsilon$ Martensitic Transformation: A Model for Stress Induced Variant and its Interaction with Grain Boundary. Journal de Physique IV Proceedings, 1995, 05 (C8), pp.C8-433-C8-438. 10.1051/jp4:1995865 . jpa-00254114

\author{
HAL Id: jpa-00254114 \\ https://hal.science/jpa-00254114
}

Submitted on 1 Jan 1995

HAL is a multi-disciplinary open access archive for the deposit and dissemination of scientific research documents, whether they are published or not. The documents may come from teaching and research institutions in France or abroad, or from public or private research centers.
L'archive ouverte pluridisciplinaire HAL, est destinée au dépôt et à la diffusion de documents scientifiques de niveau recherche, publiés ou non, émanant des établissements d'enseignement et de recherche français ou étrangers, des laboratoires publics ou privés. 


\title{
The $\gamma-\varepsilon$ Martensitic Transformation: A Model for Stress Induced Variant and its Interaction with Grain Boundary
}

\section{G. Guénin}

Institut National des Sciences Appliquées de Lyon, G.E.M.P.P.M., Bât. 502, 69621 Villeurbanne, France

\begin{abstract}
The $\gamma$ (f.c.c.) to $\varepsilon$ (h.c.p.) martensitic transformation occurs through the Shockley a/6<211> faulting every second $\{111\}$ plane of the f.c.c. structure. A stress induced thin single variant corresponds to a single a $6<211>$ faulting vector and leads to a large homogeneous shear $(0.35$ in amplitude. The tip of such a plate is composed of a set of identical Shockley partial dislocations with large mutual interactions. This work is a presentation of a model which describes the martensite morphology of stress induced $\varepsilon$ martensite in shape memory Fe-Mn-Si based alloys. The model includes the formation mechanism of the plate (Seeger's like) and its growth inside a limited grain. The mutual interaction of Shockley dislocations and their interaction with the grain boundary is semi quantitatively described; it leads to a lenticular shape of $\varepsilon$ martensite thin plates. The model is able to explain the behaviour of this kind of alloys concerning the superelastic effect and the shape memory.
\end{abstract}

\section{INTRODUCTION}

It is well known that the $\gamma$ (f.c.c.) to $\varepsilon$ (h.c.p.) martensitic transformation occurs through the a/6 $<211>$ faulting every second $\{111\}$ plane. However, the exact mechanism to obtain this faulting is not so clear. For each $\{111\}$ plane three $a / 6<211>$ vectors are possible; one single variant can therefore be defined as the result of the action of the same faulting vector every second plane, which lead to a net homogeneous shear of 0.35 . In thermal martensite, self accomodating groups of three thin variants have been observed which correspond to the same $\{111\}$ plane and the three possible a/6<211> faulting vectors in the plane [1]. Another situation has been observed which corresponds to the action of the three possible a/6 $<211>$ alternately on every second plane [2]; no net shear is therefore observed even at a very low scale. The formation mechanism of such martensite plates is not clear in spite of the existence of some models $[3,4,5]$. In the case of martensite induced by a stress, one variant is especially favoured which corresponds to a given $\{111\}$ plane associated to a single a/6 $<211>$ vector. A possible mechanism of formation of such a variant has been described by Seeger [6]. The present model uses such a mechanism in correlation to the interaction with some obstacle like a grain boundary. The model describes the shape and the behaviour of a martensite plate.

\section{2- THE MODEL}

The starting point for the stress generation of a h.c.p. martensite plate inside a f.c.c. matrix should be a mechanism analogous to the Seeger's model [6] which involves for example a screw perfect dislocation with $b_{0}=a / 2$ [211] and an imperfect dislocation with $b=a / 6$ [211] lying in the (111) plane (this imperfect dislocation may result from a Shockley dissociation). The screw perfect dislocation is supposed to be fixed and the imperfect one is supposed to be mobile due to the application of the external stress. The initial situation is shown on the figure 1 . The interaction of the two dislocations leads to the Seeger's like model of figure $2:$ the semi dislocation 1 rotates clockwise and progresses down by two (111) plane distances for each complete rotation. The semi dislocation 2 rotates anti clockwise and progresses up by two (111) plane distances for each complete rotation, therefore the f.c.c. to h.c.p. transformation occurs. 

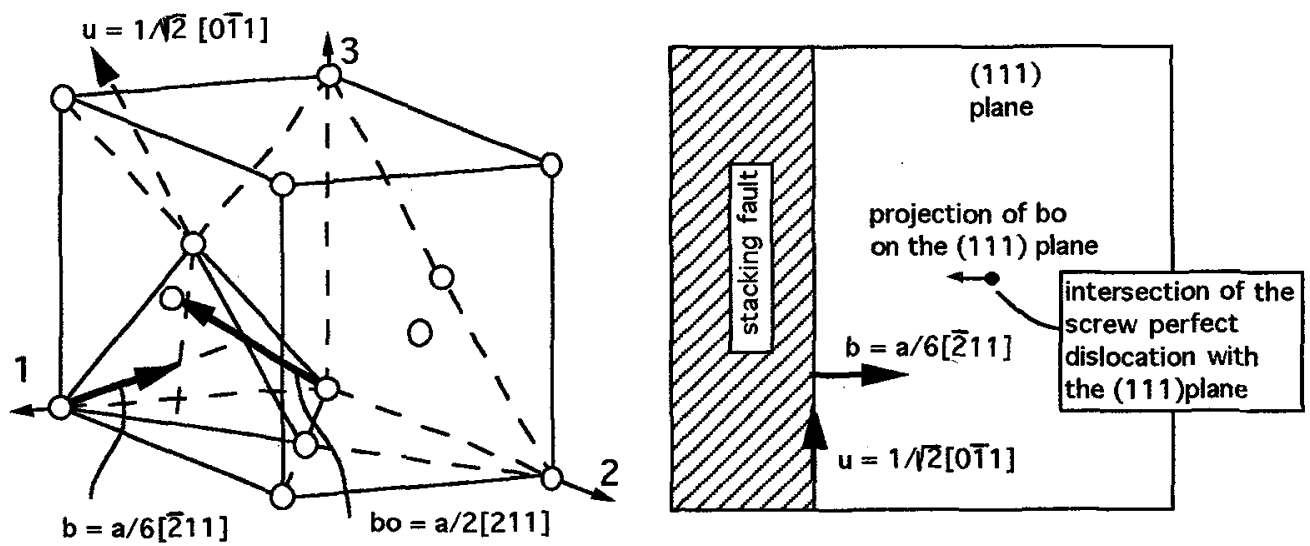

figure 1 : left, f.c.c. structure showing the different vectors involved $u$ initial line vector of the moving imperfect dislocation $\mathrm{b}$ Burgers vector of the imperfect dislocation bo Burgers vector of the perfect screw dislocation right, geometry of the problem in the (111) plane
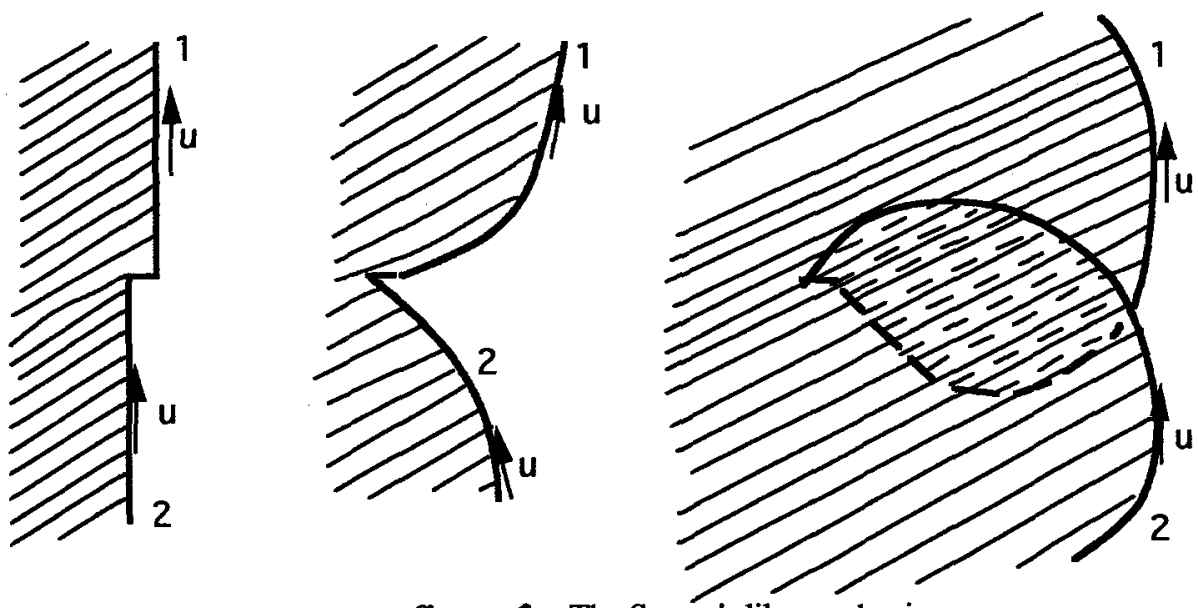

figure 2 : The Seeger's like mechanism

The real situation must take into account the fact that the crystal is limited by grain boundaries. These grain boundaries cannot be, in general situation, crossed by the mobile imperfect dislocations : there is a strong interaction between the dislocations( the h.c.p. plates) and the grain boundaries which therefore limitates the increase of the plate initiated by the previous mechanism.

Let us imagine the initial situation of a circular limited crystal with the screw dislocation in the middle and an imperfect dislocation pinned at the boundaries of the crystal (figure 3a). When the Seeger source is activated, the situation is qualitatively described by the figure $3 \mathrm{~b}$. This situation corresponds 


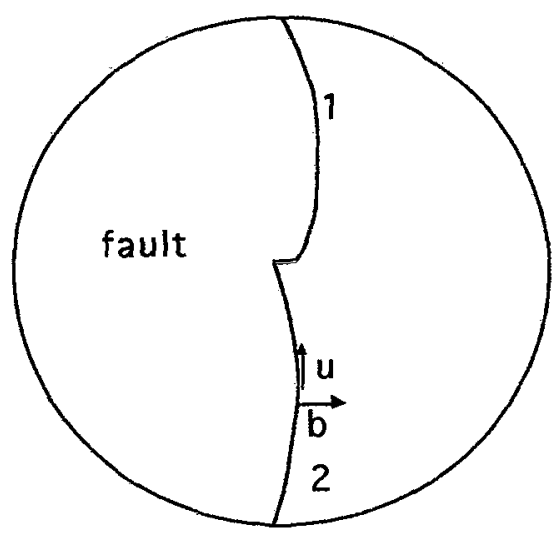

$\mathbf{a}$

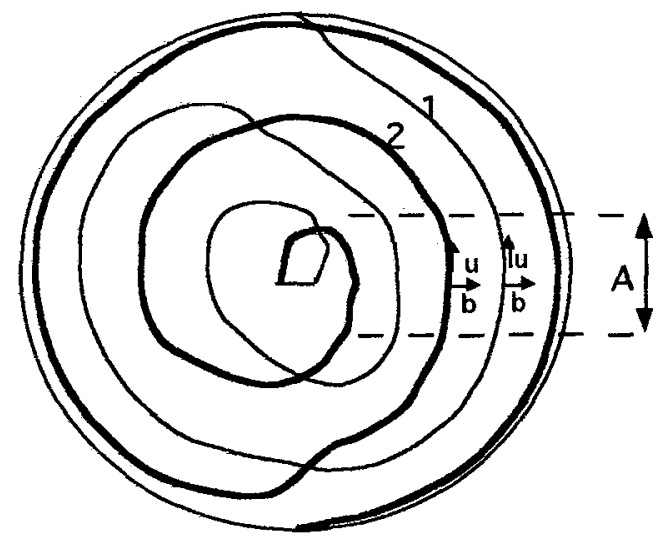

b

figure 3 : Geometry of the source in a limited circular crystal with a screw perfect dislocation in the middle and an imperfect dislocation pinned at the boundaries of the crystal

to a lenticular shape of the h.c.p. plate (figure 4). This shape depends on the interaction of the imperfect dislocations with the grain boundary and the mutual interaction of the imperfect dislocations. It is clear that for a given temperature ( fixed driving force of the transformation ) and fixed stress, these interactions limitate the thickness of the plate.

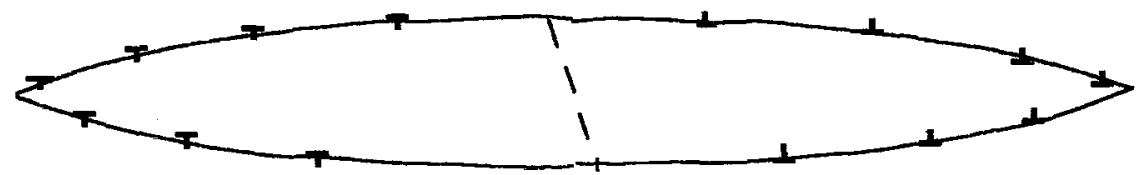

figure 4 : Lenticular shape of $\varepsilon$ martensite corresponding to the figure 3

Simplified quantification of the interactions :

Let us consider the portion A (figure 3b). Far from the source, the dislocations can be considered as parallel ones. A typical section is shown on figure 5 . Due to the geometry of the dislocation generation, the dislocations are alternately on each side of the initial fault plane. Each dislocation has a repulsive interaction with the grain boundary and with other disiocations; moreover each dislocation is

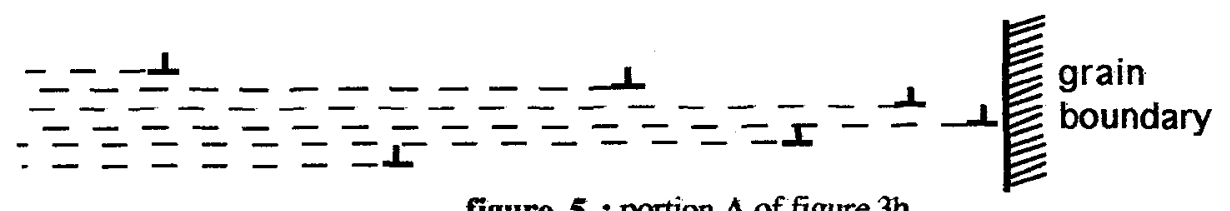

figure 5 : portion $A$ of figure $3 b$

submitted to a constant force per unit length due on one hand to the stacking fault energy $\delta$ (this energy is negative when the h.c.p. phase is more stable than the f.c.c. one) and on the other hand to the applied extemal stress, these two forces are algebraically added. The exact resolution of such a problem is rather complicated for at least two reasons : the mutual interaction of the dislocations is not simple due to the fact that they are not in the same slip plane and the interaction with the grain boundary is not easy to quantify. An approximate solution can be calculated if it is supposed that the dislocations has only relatively short range interaction with the grain boundary and if it is supposed that the horizontal 


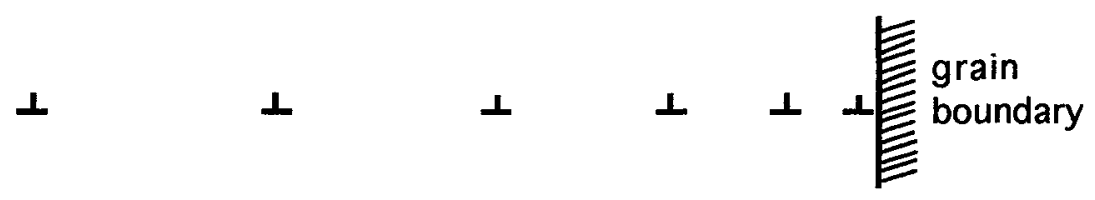

figure 6 : Simplified solution(dislocation pile up)

distances between the dislocations are large in relation to the vertical ones ( two (111) plane distances). The problem is therefore reduced to a dislocation pile up as described on figure 6 where only the front dislocation has significant interaction with the grain boundary. The unit length dislocation interaction between two consecutive dislocations is given by:

$$
\begin{aligned}
& F=\frac{\mu b^{2}}{2 \Pi(1-v)} \quad \frac{1}{--} \\
& \text { instead of } \\
& F=\frac{\mu b^{2}}{2 \Pi(1-v)} \frac{d\left(d^{2}-e^{2}\right)}{\left(d^{2}+e^{2}\right)^{2}}
\end{aligned}
$$

with $\quad b=$ Burgers vector of the partial dislocations

$\mu=$ shear modulus

$v=$ Poisson's ratio

$\mathrm{d}=$ dislocations horizontal distance

$\mathrm{e}=$ two (111) plane spaces

when $\mathrm{e}<<\mathrm{d}$ the relative error introduced by the approximation is $3 \mathrm{e}^{2} / \mathrm{d}^{2}$

This simplified situation can easily be calculated in the case of 3 dislocations. The extension to more dislocations needs some simple computer calculation. The table 1 lists the dislocation distances as a function of dislocation numbers from 3 to 6. For many dislocations, an approximate solution have been proposed by Eshelby et al [7]. In particular, the shortest separation between the first dislocation (close to the grain boundary) and the second is given by :

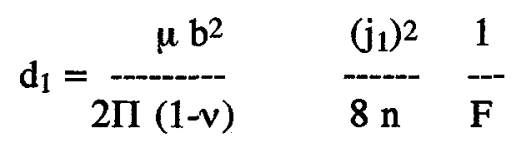

with $\mathrm{j}_{1}=$ the first zero of the Bessel function $\mathrm{J}_{1}$

$$
\mathrm{n}=\text { the dislocation number }
$$

$\mathrm{F}=$ the force per unit length applied on each

dislocation (due to stress and stacking fault energy)

For example with

$\mathrm{n}=10$, an external equivallent stress of $200 \mathrm{MPa}, \mu=70 \mathrm{GPa}$, a value of about $30 \mathrm{~b}$ is encountered for $d_{1}$ which must be compared to about $2 b$ for the e value. In these conditions the relative error introduced by the approximation is $1.3 \%$. 
As the other distances between the dislocations increases relatively fast (see table 1), the present approximate description seems to be reasonable.

Table 1 - Distances between dislocations corresponding to the pile up of figure 6 for 3 to 6 dislocations. The unit is $\mu b^{2} / 2 \operatorname{IIF}(1-v)$; $d_{i}$ is the distance between dislocations $i$ and $i+1$.

\begin{tabular}{|l|c|c|c|c|c|c|}
\hline & $\mathrm{d} 1$ (equ.3) & $\mathrm{d} 1$ & $\mathrm{~d} 2$ & $\mathrm{~d} 3$ & $\mathrm{~d} 4$ & $\mathrm{~d} 5$ \\
\hline 3 dislocations & 0.611 & 0.634 & 1.732 & & & \\
\hline 4 dislocations & 0.458 & 0.467 & 1.182 & 2.218 & & \\
\hline 5 dislocations & 0.367 & 0.372 & 0.915 & 1.581 & 2.623 & \\
\hline 6 dislocations & 0.306 & 0.309 & 0.748 & 1.251 & 1.900 & 2.935 \\
\hline
\end{tabular}

\section{3- DISCUSSION}

\subsection{Morphology of the stress induced martensite}

The present mechanism when repeated inside a grain will give a morphology schematically represented on figure 7 : the same process as the one described above occurs several times on the same \{111\} plane and with the same fault vector. This is due to the fact that the Schmidt factor of this activated system is the most favoured by the external stress. The thickness of each individual plate is correlated with the distances between the imperfect dislocations and is related to their mutual interaction and interaction with the grain boundaries as described in the present model. In the model the line tension has been neglected but qualitatively it will flatten the lenticular shape of the individual plate. Another "flattening" factor is the interaction between the plates due to the repulsive interaction of the interfaces made of dislocations of the same sign.

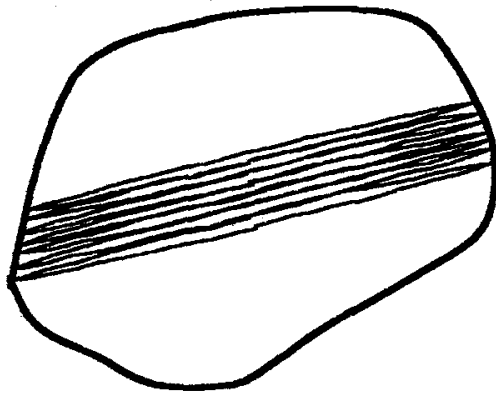

figure 7 : Schematic representation of $\varepsilon$ stress induced martensite

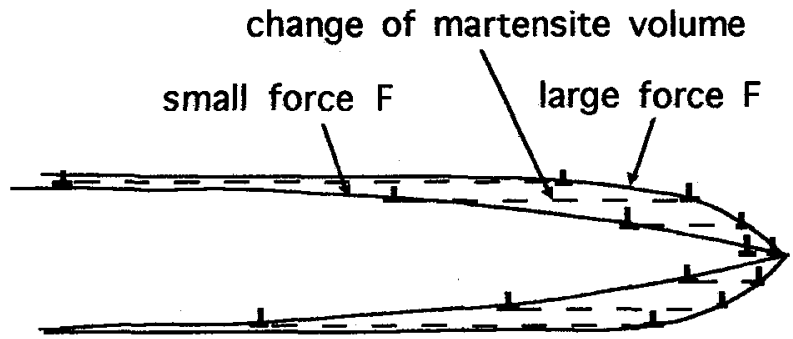

figure 8 : Change of single $\varepsilon$ martensite plate with change of force $F$

Such a morphology has been observed in stress induced martensite in a Fe-Mn-Si-Cr-Ni alloy [8]. It must be emphasized that the temperature induced martensite has a quite different morphology [8].

\subsection{The existing driving forces and the influence on the thermomechanical properties}

In the present description, two forces act as driving forces of the transformation : The chemical force and the force due to the external stress

The chemical force is related to the free energy difference between the the $\gamma$ phase and the $\varepsilon$ one. This chemical force can be described by the stacking fault energy per unit surface which is also the 
force per unit length acting on the imperfect dislocation. The sign of this force depends on the temperature in relation to $\mathrm{T}_{0}$ where no free energy difference exist between the two phases; its amplitude depends on the temperature difference $\mathrm{T}_{\mathrm{o}}-\mathrm{T}$. In the case of $\mathrm{Fe}-\mathrm{Mn}-\mathrm{Si}-\mathrm{Cr}-\mathrm{Ni}$ alloy [10] this force per unit length can be evaluated to $0.710^{-2} \mathrm{~N} / \mathrm{m}$ when the temperature is $70 \mathrm{~K}$ below $\mathrm{T}_{\mathrm{o}}$. The equivalent external stress which gives the same force per unit length is about $50 \mathrm{MPa}$.

When an external stress is applied the corresponding force on the imperfect dislocations is algebraically added. The resulting force is the one involved in the distances between the dislocations (equation (3), table 1). It can be seen that these distances change as 1/F. Even if the exact situation is sligthly different due to the additional interactions, the qualitative situation can be described by the figure 8 when the force $F$ increases or decreases the plate beeing already present.

Action of a stress at a temperature above $M_{S}$ and below $T_{0}$ :

The chemical driving force is insufficient to induce the nucleation of the transformation, but the application of the external stress induces the nucleation and growth of plates as described in the model. The growth is limited by the interaction of the plates with the grain boundaries. When the external stress is removed, the chemical force only remains leadind to a reverse movement of the dislocations (i.e. interfaces as described on figure 8). This reversible movement of the interfaces must lead to a superelastic effect. Such an effect has already been observed by Yang et al [9] on Fe-Mn-Si based alloys.

The shape memory :

When no external stress is applied after the upper experiment, the martensite volume is only controlled by the chemical force (stacking fault energy). When the temperature is increased, the fault energy decreases (becomes less negative) leading to a decrease of the martensite volume that is to say a strain recovery. This recovery occurs just above the temperature of the stress application. This is in agreement with the experimental observations : the strain recovery indeed begin to occur immediately by heating above the temperature of the stress application and not at the As temperature corresponding to the thermally induced martensite [10]. In this hypothesis the present mechanism could therefore be responsible for the shape memory effect in such a kind of alloys. The fact that the strain recovery (i.e. martensite vanishing) generally spreads above $T_{0}$ and even $\mathbf{A}_{\mathbf{f}}$ temperature (defined for thermal martensite) should be due to the interaction of the "usefull" imperfect dislocations with other "bad" dislocations : the stacking fault energy must become positive to favour the imperfect dislocations come back.

\section{4- CONCLUSION}

The present model of stress induced $\varepsilon$ martensite in a $\gamma$ matrix, based on a Seeger's mechanism in association with grain boundary interaction is able to explain qualitatively a lot of experimental observations :

The morphology of the stress induced martensite

The superelastic effect

The origin of the strain recovery and its temperature behaviour (shape memory effect)

\section{References}

[1] Yang J.H. and Wayman C.M.,Acta metall. mater. 40 (1992) 2011-2023 and 2025-2031

[2] Puteaux J.L., Federzoni L., Mantel M., Blanc G. and Chevalier J.P., "EUROMAT 93"J. de phys. 4(1994) 175-180

[3] Lysak L.I., Fiz. metal. metall. 20 (1967) 547-554

[4] Delamotte E. and Altstetter C., Trans. AIME. 245 (1969) 651-659

[5] Fujita H. and Ueda S., Acta met. 20 (1972) 759-767

[6] Seeger A., Z. Metallkunde 46 (1956) 653-660

[7] Eshelby J.D., Frank F.C. and Nabarro F.R.N., Phil. mag. 42 (1951) 351-364

[8] Bergeon N. and Guénin G., present conference

[9] Yang J.H., Chen H. and Wayman C.M., Metall. Trans. 23A (1992) 1431-1437

[10] Federzoni L., Thesis, Institut National des Sciences Appliquées de Lyon (1993) 